\title{
Research on Dynamic Coverage Based on V-DE Algorithm in Wireless Sensor Networks
}

\author{
Y.X. Zhang, X.Y. Li \\ School of Electronics and Information Engineering \\ Beijing Jiaotong University \\ Beijing, China
}

\begin{abstract}
With the development and application of sensor network technology, how to achieve a dynamic multi-objective sensor network coverage optimization has become an important issue currently facing the sensor network. By analysis of the original algorithm for the dynamic overlay node mobility departure, VDE algorithm was put forward to optimize the degree of coverage which combines the advantages of both differential evolution algorithm and virtual force algorithm. The simulation results have shown that the derived virtual force-differential evolution algorithm has a significant effect optimization on the stability of dynamic coverage.
\end{abstract}

Keywords-dynamic coverage; wireless sensor networks; v-de algorithm

\section{INTRODUCTION}

Wireless sensor networks [1] are built on the development of the micro electro mechanical system, system-on-chip, wireless communication and low power embedded technology, which are very convenient and economical in a practical environment. Wireless sensors are applied to a wide range of areas.

Network coverage [2], which is about the deployment of sensor network nodes to achieve the maximization of network coverage, was one of the fundamental problems in wireless sensor networks when we construct them.

At present, the control algorithms in the wireless sensor network coverage usually is the particle swarm optimization algorithm [3] (PSO), genetic algorithm [4] (GA), ant colony algorithm [5] (ACO), virtual force algorithm [6] (VF) and so on.

\section{PROBLEM DESCRIPTION}

\section{A. Sensor Node Model}

In the actual modeling, the sensing model of the sensor nodes is the Boolean sensing model [7], as shown in Fig 2-1. Considering the coverage area of the sensor nodes as a circular area which the center is the sensor nodes and the radius is $R_{s}$, we put the circular area known as the sensing scope of the node, and the radius known as the sensing radius of the sensor nodes. The sensing radius of the sensor nodes depends on the type of sensor nodes. Assume the coordinate of the position of the sensor nodes $\mathrm{s}$ is $\left(x_{s}, y_{s}\right)$. For any point $\mathrm{p}$ in a plane, its

\author{
Z.Q. Huang \\ School of Software \\ Beijing University of Technology \\ Beijing, China
}

location is $\left(x_{p}, y_{p}\right)$. And the probability of the node $\mathrm{s}$ detecting an arbitrary point $\mathrm{p}$ is

$$
C(s, p)=\left\{\begin{array}{l}
1 \quad d(s, p) \leq R_{s} \\
0 \quad \text { otherwise }
\end{array}\right.
$$

In the formula

$$
\begin{gathered}
d(s, p)=\sqrt{\left(x_{z}-x_{p}\right)^{2}+\left(y_{s}-y_{p}\right)^{2}} \\
d(s, p) \text { is the Euclidean distance from node } \mathrm{p} \text { to node } \mathrm{s} \text {. }
\end{gathered}
$$

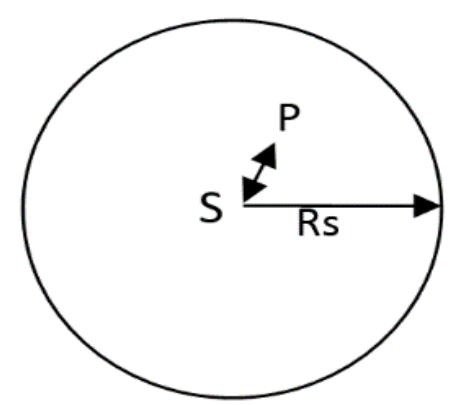

FIGURE 1. BOOLEAN SENSING MODEL.

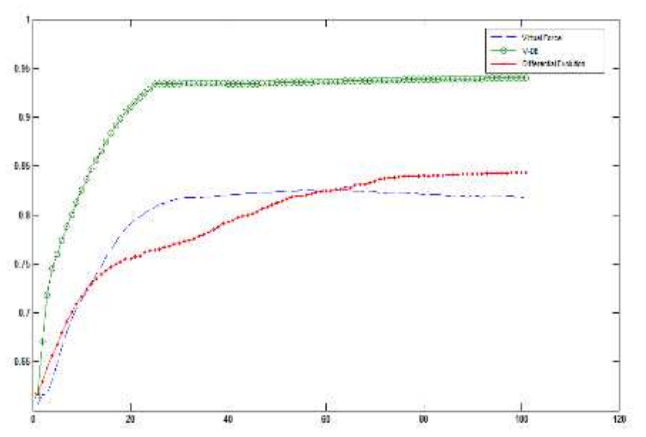

FIGURE 2. COVERAGE RATE CURVE.

\section{B. Coverage Rate}

Coverage rate has direct relation with the network's degree of sensing. A good coverage rate can make the utilization of space resources in wireless sensor networks maximal.

Consider that the region has k nodes $S_{1}, S_{2}, \cdots, S_{k}$, their coverage to point $\mathrm{p}$ respectively are 


$$
C\left(s_{1}, p\right), C\left(s_{2}, p\right), \cdots, C\left(s_{k}, p\right) .
$$

Coverage at point $\mathrm{p}$ is

$$
C_{p}=1-\prod_{i=1}^{k}\left(1-C\left(s_{i}, p\right)\right)
$$

The coverage rate of wireless sensor networks are defined as the ratio of the sum of the coverage area of all nodes and the area of the entire target area.

$$
C=\bigcup_{i=1}^{k} \frac{S_{i}}{S} .
$$

\section{NODE DEPLOYMENT BASED ON V-DE ALGORITHM}

\section{A. Virtual Force Algorithm}

The virtual force algorithm [8] assumes the whole mobile sensor network is a virtual physical system. The virtual system contains the attraction or repulsion that both the sensor node and the regional nodes are applied to the sensor. The moving direction and speed of the node depend on the resultant force of the attraction and repulsion of neighboring nodes.

Repulsion from neighboring nodes

Assume arbitrary two nodes $S_{i}$ and $\mathrm{S}_{j}$, node $S_{i}$ is under the repulsion of ${ }^{S_{j}}$. It meets the following relation:

$$
F_{i j}\left(s_{i}, s_{j}\right)=\left\{\begin{array}{cc}
\frac{k_{1} m_{i} m_{j}}{d\left(s_{i}, s_{j}\right)^{a_{1}}} & 0<d\left(s_{i}, s_{j}\right) \leq r_{s}\left(s_{i}\right) \\
0 & d\left(s_{i}, s_{j}\right) \geq r_{s}\left(s_{i}\right) .
\end{array}\right.
$$

In the formula, $k_{1}$ and $a_{1}$ are gain coefficients, $m_{i}$ and $\mathrm{m}_{j}$ is the mass of the nodes. In this paper, take the square of the node sensing radius as the mass. $d\left(s_{i}, s_{j}\right)$ is the distance between $S_{i}$ and $\mathrm{S}_{j}$.

\section{Attraction from uncovered area}

Assume node $S_{i}$ is under the attraction that Npot exerts on. The attraction is expressed as:

$$
F_{\text {ihot }}\left(s_{i}, N p o t\right)=\frac{-k_{2}}{d\left(s_{i}, N p o t\right)^{a_{2}}} .
$$

In the formula, $k_{2}$ and $a_{2}$ are gain coefficients, $d\left(s_{i}, N p o t\right)$ is the distance between $s_{i}$ and Npot.

\section{Stress analysis of nodes}

In the virtual physical system of the virtual force, nodes will move to a new location $\left(x_{n}, y_{n}\right)$ from the original location $\left(x_{o}, y_{o}\right)$ under the effect of virtual force.

$$
\begin{gathered}
x_{n}=\left\{\begin{array}{cl}
x_{o} & , F_{X Y}<F_{t h} \\
x_{o}+\frac{F_{X}}{F_{x y}} \times \text { Maxstep } \times e^{-\frac{1}{F_{x y}}} & , F_{x y} \geq F_{t h}
\end{array}\right. \\
y_{n}=\left\{\begin{array}{cl}
y_{o} & , F x y<F_{t h} \\
y_{o}+\frac{F_{y}}{F_{x y}} \times \text { Maxstep } \times e^{-\frac{1}{F_{x y}}} & , F_{x y} \geq F_{t h}
\end{array}\right.
\end{gathered}
$$

In the formula, $F_{\mathrm{xy}}$ is the virtual resultant force that the system exerts on sensor nodes, and $F_{\mathrm{x}}, F_{\mathrm{y}}$ is respectively the component of $\mathrm{X}$ axis and $\mathrm{Y}$ axis. $F_{\text {th }}$ is the threshold of virtual force. If the virtual resultant force of the sensor node is less than this value, the sensor node will not generate the moving acceleration, that is to say, it will not produce a mobile sensor node. Or else, it will generate motion under the effect of virtual force. The speed and direction of movement depend on the force and its mass. Maxstep is the maximum moving distance of the sensor node.

\section{B. Differential Evolution Algorithm}

Differential evolution algorithm [9] is an evolutionary algorithm based on population. The basic principle of algorithm is making use of the operation of mutation and crossover to produce new generation of the population, and then producing optimal population through the operation of selection between two generations.

The strategy of the differential evolution algorithm can effectively optimize the layout of wireless sensor networks. However, since the complexity of the differential evolution algorithm will increase exponentially with expanding of the optimized vector dimension[10], the computation time has been one of the bottlenecks for the DE algorithms to solve in the layout optimization of the wireless sensor networks.

\section{The Basic Principle of V-De Algorithm}

Since both the virtual force algorithm and the DE algorithm have their own shortcomings in the process of deploying nodes, we propose the V-DE algorithm according to the characteristic of them. The basic principle is as follows.

(1)Set the initial parameters. Deploy a plurality of sensor nodes randomly in the designated area, and then calculate the coverage rate $\mathrm{c}_{0}$ of the initial state.

(2)Analysis of the force on each node in accordance with the formula (3-1) and formula (3-2), and then get the new position $\left(\mathrm{x}_{\mathrm{i}}, \mathrm{y}_{\mathrm{i}}\right)$ of each node according to the formula (3-3) and the formula (3-4).Set $\delta_{i}=\nabla C_{i} / \nabla C_{i-1}$, and calculate the current coverage rate $c_{i}$ and coverage rate gradient $\delta_{i}$.

(3)Compare $\delta_{\mathrm{i}}$ and $\delta_{t h}$. If $\delta_{i} \geq \delta_{t h}$, update the node positions and repeat step 2, otherwise, retain the position $\left(x_{i-1}, y_{i-1}\right)$ of each node and perform Step 4.

(4)Set the current positions of the nodes as the initial population, and set the scaling factor $\mathrm{F}$ of the population, the 
maximum number maxiter of iterations and crossover probability factor $\mathrm{CR}$.

(5)Assuming the iteration number is $i$, the population is initialized. Select three individuals $g_{r 1}(i), g_{r 2}(i), g_{r 3}(i)$ from the parent population as the mutation components. The mutation operation is as follows

$$
V_{r}(i+1)=g_{1 r}(i)+F \cdot\left(g_{2 r}(i)-g_{3 r}(i)\right)
$$

In the formula, $V_{r}(i+1)$ is the new individual of the $i+1$ th generation that is mutated from the $\mathrm{i}$-th generation.

(6)The crossover is based on Binomial Distribution according to CR:

$$
u_{r}(i+1)=\left\{\begin{array}{cl}
v_{r}(i+1) & , \text { rand }<C R \\
V_{r}(1) & , \text { rand } \geq C R .
\end{array}\right.
$$

In the formula, $u_{r}(i+1)$ is the new individual of the $i+1$ th generation that is crossed from the $\mathrm{i}$-th generation. rand is a uniform random number between 0 and 1 .

(7)The selection is to compare the parent and offspring, and their relationship is as follows:

$$
g_{r}(i+1)=\left\{\begin{array}{cl}
u_{r}(i) & , C_{i}<C_{i+1} \\
u_{r}(i+1) & , C_{i} \geq C_{i+1} .
\end{array}\right.
$$

Among them, $C_{i}$ is the selection function, and $g_{r}(i+1)$ is the new individual of the $i+1$ th generation that is selected from the $\mathrm{i}$-th generation.

(8)Judge about the iteration time. If it's not over the maxiter, set $\mathrm{i}=\mathrm{i}+1$, and repeat Step 5,otherwise,finish the circulation.

\section{A. Simulation Experiments and Results Analysis}

The experiment of the V-DE algorithm is conducted by MATLAB. In the $1100 \times 1100(\mathrm{~m} 2)$ square area 40 randomly distributed sensors are arranged, sensors' types are set to 1, the radius of sensors sensing is set to $90(\mathrm{~m})$. Communication radius is twice the radius of sensing. The maximum iterations number is 100 times. Ignoring the mobile node energy consumption during exercise, connectivity is considered to be intact. The results of simulation experiments is shown below.
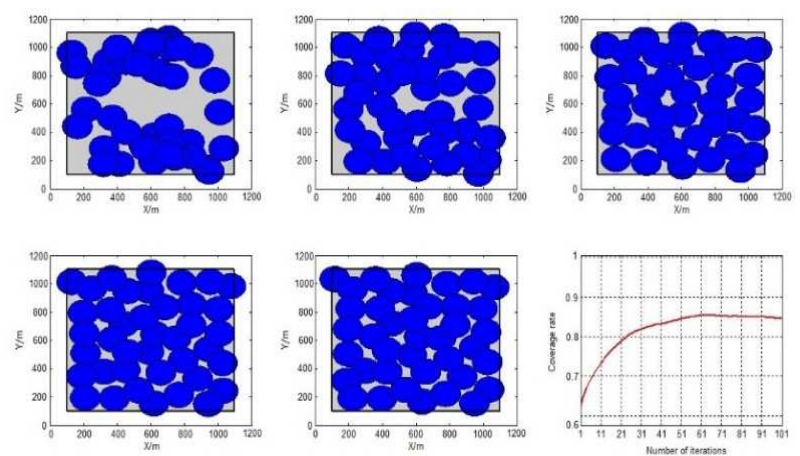

FIGURE 3. NODE DISTRIBUTION STATE DIAGRAM AND COVERAGE RATE CURVE.
Figure 3-1 shows the node distribution state and coverage rate curve. The first picture is the positions of the initial state, and it is the distribution state of the nodes' positions of the 25 th times, 50th times, 75th times, 100th times in turn from the 2nd picture to the 5th picture. The 6th picture is the coverage rate graph, where the horizontal axis is the number of iterations, and the vertical axis is the network coverage. There is a significant shock on the coverage of the curve. Iterative process in the transition between the two algorithms may exist a phenomenon of the node local convergence, leading to a smaller fall in the coverage curve.

Figure 3-2 is the coverage rate graph for the three algorithms in the same situations, where the horizontal axis is the number of iterations, and the vertical axis is the network coverage. It is easily to make a conclusion that to meet the connectivity conditions, the coverage rate reaches $93 \%$ by the V-DE algorithm which is improved obviously than the other two algorithms. The V-DE algorithm meets the basic task of effective coverage. The tortuous phenomenon in the node moving route is reducing significantly comparing with the virtual force algorithm. The number of the iteration time to reach the maximum coverage rate is lower than the DE algorithm. Therefore, the V-DE algorithm not only solves the problem of post-concussion in the virtual force algorithm, but also make ups for the defects of the pre-high computational complexity in the DE algorithm.

\section{CONCLUSION}

The node deployment in the wireless sensor networks determines the performance of the network coverage, and directly affects the network information collection and transmission. This paper studies the advantage and disadvantage of the virtual force algorithm and the DE algorithm relying on dynamic sensor node deployment. Through the comparative analysis, the V-DE algorithm is proposed on the basis of the two algorithms above. The V-DE algorithm consists of the advantages of them, preserving the rapid convergence of the virtual force algorithm and the optimization characteristics of the DE algorithm. Therefore, the V-DE algorithm has a better performance in solving the problem of network coverage control in wireless sensor networks.

\section{ACKNOWLEDGEMENTS}

This work is supported by the Fundamental Research Funds for the Central Universities (KWJB13042536).

\section{REFERENCES}

[1] Ren Feng-yuan, Huang Hai-ning, Lin chuang. Wireless Sensor Networks. Journal of Software, 2003, 14(7): 1282-1291.

[2] WANG Wei, LIN Feng, ZHOU Ji-liu. Research progress on coverage problem in wireless sensor network. Application Research of Computers, 2010, 27(1)

[3] XIANG Xi-xi, HUANG Hong-guang, LI Yu-dong. Hybrid sensor networks coverage-enhancing approach based on particle swarm optimization. Application Research of Computers, 2010, 27(6): $2273-$ 2275.

[4] ZHONG You-ping, HE Wei-li, WANG Guang-huang. WANG Zi-zhan, Coverage optimization based on gene algorithm $(G A)$ in wireless sensor network. Information Technology, 2011 (6): 18-23.

[5] WANG Ying, XIE Jian-ying. An Adaptive Ant Colony Optimization 
Algorithm and Simulation. Acta Simulata Systematica Sinica, 2005 (1): 31-33.

[6] Zhou Tong, Hong Bing-rong, and Piao Song-hao. Hybrid Sensor Networks Deployment Based on Virtual Force. Journal of Computer Research and Development, 2007, 44(6): 965-972

[7] MA Hua-Dong+, TAO Dan. Multimedia Sensor Network and Its Research Progresses. Journal of Software, 2006, 17(9): 2013-2028.

[8] Tan G, Jarvis S A, Kermarrec A M. Connectivity-guaranteed and obstacle-adaptive deployment schemes for mobile sensor networks. Mobile Computing, IEEE Transactions on, 2009, 8(6): 836-848.

[9] Wang B, Lim H B, Ma D. A survey of movement strategies for improving network coverage in wireless sensor networks. Computer Communications, 2009, 32(13): 1427-1436.

[10] LIU Bo, WANG Ling, JIN Yi-hui. Advances in differential evolution. Control and Decision, 2007, 22(7): 721-729. 